\title{
On Fast Computation of Directed Graph Laplacian Pseudo-Inverse
}

\author{
Daniel Boley \\ University of Minnesota \\ boley@umn . edu
}

September 16, 2020

\begin{abstract}
The Laplacian matrix and its pseudo-inverse for a strongly connected directed graph is fundamental in computing many properties of a directed graph. Examples include random-walk centrality and betweenness measures, average hitting and commute times, and other connectivity measures. These measures arise in the analysis of many social and computer networks. In this short paper, we show how a linear system involving the Laplacian may be solved in time linear in the number of edges, times a factor depending on the separability of the graph. This leads directly to the column-by-column computation of the entire Laplacian pseudo-inverse in time quadratic in the number of nodes, i.e., constant time per matrix entry. The approach is based on "off-the-shelf" iterative methods for which global linear convergence is guaranteed, without recourse to any matrix elimination algorithm.
\end{abstract}

Keywords:. Graph Laplacian; Directed Graphs; Pseudo-Inverse; Iterative Methods.

\section{Introduction.}

Many properties of networks can be found via the solution of special linear systems based on the graph Laplacian. Examples include the well-known pagerank, centrality measures, betweenness measures, graph cuts, distances or affinities between nodes, trust/influence propagation, etc. [5, 8, 14, 17, 27, 38]. These properties have spawned many papers on efficient, almost linear time solvers for these special linear systems such as [36] for symmetric systems for undirected graphs to more recent papers reporting almost linear time for non-symmetric Eulerian Laplacians for directed graphs [10, 11]. For the purposes of this paper, we say a Laplacian matrix $\mathbf{L}$ is "Eulerian" if $\mathbf{L}$ has nullity 1 and $\mathbf{L w}=\mathbf{L}^{T} \mathbf{w}=\mathbf{0}$ for a strictly positive vector $\mathbf{w}$. These fast methods use a careful ordering of the nodes, an approximate factorization using Gaussian elimination used as a preconditioner to an iterative method based on, e.g., Richardson iteration. The theoretical running time for the methods of [10, 11] can be bounded by $O(m) \log ^{c}(n \kappa \varepsilon)$ with high probability $(1-\delta)$, where $m$ is the number of edges, $n$ the number of nodes, $\kappa$ is the matrix condition number, and $\varepsilon$ is the desired accuracy, with $O(n) \varepsilon^{-2} \log ^{c}(1 / \delta) \log ^{c}(n \kappa \varepsilon)$ fill-in from the inexact factorization. The $c$ 's are some arbitrary constants. In this short paper we use a different approach to obtain an algorithm for the pseudo-inverse of a non-symmetric Eulerian Laplacian. Our approach is to use only iterative methods in widespread use in practice, and which also enjoy provable linear convergence guarantees and per-iteration costs linear in the number of edges in the graph. We also propose a computational process to obtain an Eulerian scaling. By using only iterative methods, we avoid the issue of fill-in entirely. This paper focuses on strongly connected directed graphs. The results carry over to the case of connected undirected graphs, but most of the results can be simplified. This is beyond the scope of this paper.

The rest of this paper begins with preliminaries to introduce the Laplacians and other basic matrices associated with directed graphs, followed by a theorem which reduces the pseudo-inverse computation to 
a simple matrix inversion. Then we present the overall algorithm to find an Eulerian scaling and compute the pseudo-inverses for Eulerian Laplacians, followed by an outline of the complexity analysis, which includes the convergence theory and the cost per iteration. We then briefly show how the pseudo-inverse for a non-Eulerian Laplacian can be recovered from that of an Eulerian Laplacian. We end with a short table of experiments showing the performance of the methods in practice is consistent with the theoretical complexity bounds. We collect existing theoretical results on which our methods are based into an Appendix. Throughout this paper, all norms are the matrix or vector 2-norms, unless otherwise specified.

2. Preliminaries. Consider a directed graph with adjacency matrix $\mathbf{A} \in \mathbb{R}^{n \times n}$ where $a_{i j}$ is the weight on the edge $i \rightarrow j$ if such an edge exists, otherwise $a_{i j}=0$. If $\mathbf{1}$ is the vector of all ones of appropriate dimension, then $\mathbf{d}=\mathbf{A} \mathbf{1}$ is the vector of out-degrees, $\mathbf{D}=\operatorname{Diag}(\mathbf{d})$ is the diagonal matrix with the entries of $\mathbf{d}$ on the diagonal, and $\mathbf{P}=\mathbf{D}^{-1} \mathbf{A}$ is the matrix of transition probabilities for a random walk over this directed graph. Throughout this paper we assume the graph is strongly connected implying that $\mathbf{P}$ is irreducible. Let $\pi$ be the unique vector of stationary probabilities over this graph, i.e., the vector satisfying $\boldsymbol{\pi}^{T} \mathbf{P}=\boldsymbol{\pi}^{T}$ and $\boldsymbol{\pi}^{T} \mathbf{1}=\mathbf{1}$, and let $\boldsymbol{\Pi}=\operatorname{Diag}(\boldsymbol{\pi})$ be the diagonal matrix with the stationary probabilities $\left\{\pi_{i}\right\}_{1}^{n}$ on the diagonal. Perron-Frobenius theory guarantees $\pi$ exists and is strictly positive [15, 21]. Several different Laplacians have been defined for a given digraph, each related to each other through a variety of diagonal scalings [5]:

$$
\begin{array}{lll}
\mathbf{L}^{\mathrm{r}}=\mathbf{\Pi}-\mathbf{\Pi} \mathbf{P} & \text { random walk Laplacian } \\
\mathbf{L}^{\mathrm{a}}=\mathbf{D}-\mathbf{A}=\mathbf{D}-\mathbf{D P} & \text { unnormalized Laplacian } \\
\mathbf{L}^{\mathbf{p}}=\mathbf{I}-\mathbf{P} & \text { normalized Laplacian } \\
\mathbf{L}^{\mathrm{d}}=\mathbf{I}-\mathbf{\Pi}^{1 / 2} \mathbf{P} \mathbf{\Pi}^{-1 / 2} & & \text { diagonally scaled Laplacian }
\end{array}
$$

and corresponding pseudo-inverses

$$
\mathbf{M}^{\mathrm{r}}=\left(\mathbf{L}^{\mathrm{r}}\right)^{\dagger}, \mathbf{M}^{\mathrm{d}}=\left(\mathbf{L}^{\mathrm{d}}\right)^{\dagger}, \mathbf{M}^{\mathrm{p}}=\left(\mathbf{L}^{\mathrm{p}}\right)^{\dagger}, \text { etc. }
$$

It is well known that the Laplacians are interchangeable in the limited sense that one can obtain many graph properties from one or another of the Laplacians or their pseudo-inverses, e.g., the average length $h(i, k)$ of a random walk starting from node $i$ before reaching node $k$ and the average round-trip commute time $c(i, k)$ $[2,5,14,20,23,24,25,30]$ (even for strongly connected directed graphs):

$$
\begin{gathered}
h(i, k)=\mathbf{m}_{k k}^{\mathrm{r}}-\mathbf{m}_{i k}^{\mathrm{r}}+\sum_{\ell}\left(\mathbf{m}_{i \ell}^{\mathrm{r}}-\mathbf{m}_{k \ell}^{\mathrm{r}}\right) \pi_{\ell}=\frac{\mathbf{m}_{k k}^{\mathrm{d}}}{\pi_{k}}-\frac{\mathbf{m}_{i k}^{\mathrm{d}}}{\sqrt{\pi_{i} \pi_{k}}} \\
c(i, k)=\mathbf{m}_{k k}^{\mathrm{r}}+\mathbf{m}_{i i}^{\mathrm{r}}-\mathbf{m}_{i k}^{\mathrm{r}}-\mathbf{m}_{k i}^{\mathrm{r}}=\frac{\mathbf{m}_{k k}^{\mathrm{d}}}{\pi_{k}}+\frac{\mathbf{m}_{i i}^{\mathrm{d}}}{\pi_{i}}-\frac{\mathbf{m}_{i k}^{\mathrm{d}}+\mathbf{m}_{k i}^{\mathrm{d}}}{\sqrt{\pi_{i} \pi_{k}}}
\end{gathered}
$$

The choice of which Laplacian scaling to use depends on which leads to a simpler formula.

The pseudo-inverse also yields the average number of visits to an individual node $j$ for random walks starting in node $i$ before reaching $k:$ [4, 18]:

$$
v(i, j, k)=\left(\mathbf{m}_{i j}^{\mathrm{r}}-\mathbf{m}_{k j}^{\mathrm{r}}-\mathbf{m}_{i k}^{\mathrm{r}}+\mathbf{m}_{k k}^{\mathrm{r}}\right) \pi_{j}=\sqrt{\frac{\pi_{j}}{\pi_{i}}} \mathbf{m}_{i j}^{\mathrm{d}}-\sqrt{\frac{\pi_{j}}{\pi_{k}}} \mathbf{m}_{k j}^{\mathrm{d}}-\frac{\pi_{j}}{\sqrt{\pi_{i} \pi_{k}}} \mathbf{m}_{i k}^{\mathrm{d}}+\frac{\pi_{j}}{\pi_{k}} \mathbf{m}_{k k}^{\mathrm{d}},
$$

and the probability that such a random walk passes node $j$ at all:

$$
\operatorname{Prob}(\text { pass } j \text { on walks } i \rightarrow k)=v(i, j, k) / v(j, j, k) \text {. }
$$


By summing $v(i, j, k)$ across various dimensions, one can obtain various centrality and betweenness measures for individual nodes [18]. For example, it can be easily verified that summing (4) over $j$ yields formula (3): $\sum_{j} v(i, j, k)=h(i, k)$, and summing (3) over $k$ yields $\sum_{k} \pi_{k} h(i, k)=$ trace $\mathbf{M}^{\mathrm{d}}$ (independent of $i$ ) equivalent to the Random Target Lemma [2].

Remark. To illustrate how these relations are a simple consequence of our Theorem 1 below, a derivation for the last equality in (4) is given here. A derivation had not given previously elsewhere to the author's knowledge. Apply Theorem 1 by setting $A=\mathbf{L}^{\mathrm{d}}=\boldsymbol{\Pi}^{1 / 2}(\mathbf{I}-\mathbf{P}) \boldsymbol{\Pi}^{-1 / 2}, \mathbf{u}=\sqrt{\pi}$, and $B=\mathbf{M}^{\mathrm{d}}=\left(\mathbf{L}^{\mathrm{d}}\right)^{\dagger}$. Writing (7) in Theorem 1b elementwise, we have:

$$
\left[A_{11}^{-1}\right]_{i j}=\mathbf{m}_{i j}^{\mathrm{d}}-\sqrt{\frac{\pi_{i}}{\pi_{n}}} \mathbf{m}_{n j}^{\mathrm{d}}-\sqrt{\frac{\pi_{j}}{\pi_{n}}} \mathbf{m}_{i n}^{\mathrm{d}}+\frac{\sqrt{\pi_{i} \pi_{j}}}{\pi_{n}} \mathbf{m}_{n n}^{\mathrm{d}}
$$

Apply two-sided diagonal scaling $(\mathbf{I}-\mathbf{P})=\mathbf{\Pi}^{-1 / 2} A \mathbf{\Pi}^{1 / 2}$ to obtain

$$
v(i, j, n)=\left[\left(\mathbf{I}-\mathbf{P}_{11}\right)^{-1}\right]_{i j}=\sqrt{\frac{\pi_{j}}{\pi_{i}}}\left[A_{11}^{-1}\right]_{i j}=\sqrt{\frac{\pi_{j}}{\pi_{i}}} \mathbf{m}_{i j}^{\mathrm{d}}-\sqrt{\frac{\pi_{j}}{\pi_{n}}} \mathbf{m}_{n j}^{\mathrm{d}}-\frac{\pi_{j}}{\sqrt{\pi_{i} \pi_{n}}} \mathbf{m}_{i n}^{\mathrm{d}}+\frac{\pi_{j}}{\pi_{n}} \mathbf{m}_{n n}^{\mathrm{d}}
$$

Given an arbitrary directed graph with $n$ nodes, one can augment the graph with an extra node $n+1$ such that, upon every transition in a random walk over the graph, there is a small probability $\gamma$ that the walker "evaporates" to node $n+1$, and thence the walker transitions to an arbitrary node with equal probability (or biased probabilities in a personalized setting). This is a process very similar to teleportation in the pagerank setting. The result is a strongly connected directed graph to which we can apply the methods of this paper. In this case the average number of visits $v(i, j, n+1)$ or average path lengths $h(i, j)$ would yield a continuum of affinity estimates from $i$ to $j$, approximating random walk affinity for $\gamma$ near 0 and shortest path affinity for $\gamma$ near 1 . The probability $v(i, j, n+1) / v(j, j, n+1)$ of equation (5) can be interpreted as the trust of node $j$ from the point of view of node $i$ in a trust network [6, 27]. The sum $\sum_{i} v(i, j, n+1) / v(j, j, n+1)$ can be interpreted as an average level of trust in node $j$ or a measure of influence of node $j$ in a social network [17].

The main contributions of this paper are: (A) we show how an off-the-shelf iterative method in widespread use yields a method to find the pseudo-inverse of an Eulerian Laplacian with a provable complexity guarantee that is linear in the number of edges times a factor related to the connectness of the graph ; (B) we show how another off-the-shelf method yields a method to find a Eulerian scaling for a non-Eulerian Laplacian with similar complexity guarantees; and (C) we illustrate the methods with some examples showing the linear complexity can be observed in practice with the off-the-shelf numerical procedures. The constructions proposed in this paper are kept as simple as possible to highlight a minimal set of assumptions needed to form the basis for a fast Laplacian solver. Most of the theoretical properties used in this paper are well-known, but we include a few brief proofs to make this paper more self-contained.

The computation of the Moore-Penrose pseudo-inverse in the general case usually proceeds using the SVD using $O\left(n^{3}\right)$ time [19]. However, a graph Laplacian for strongly connected digraphs has nullity equal to 1 . This special property allows one to compute the pseudo-inverse with just an ordinary matrix inversion using the formulas given in theorems 1 (a), 4(a) below, based on the theory of [28], assuming one knows the left and right nullspaces for the Laplacian. The cost of the matrix inversion using a standard algorithm like Gaussian elimination is also $O\left(n^{3}\right)$ but much faster than a full SVD [19]. The cost to obtain even a single column of the pseudo-inverse using Gaussian elimination is still $O\left(n^{3}\right)$. The goal in this paper is to show how this complexity can be reduced to $O(m \cdot \log \kappa \varepsilon)$ for each column of the pseudo-inverse and to $O(n m \cdot \log \kappa \varepsilon)$ for the entire pseudo-inverse, where $m$ is the number of edges. The procedures outlined 
here also include the computation of the necessary left and right nullspaces with $O(m \cdot \log \kappa \varepsilon)$ cost. An undirected graph would lead to subtantial simplifications and often lower cost using different techniques, but this is beyond the scope of this paper.

\section{Theoretical Construction.}

In this paper we study mainly the Eulerian Eulerian Laplacian matrices $\mathbf{L}^{\mathrm{r}}$ and $\mathbf{L}^{\mathrm{d}}$ (1). The matrix $\mathbf{L}^{\mathrm{r}}$ can be thought of as the unnormalized Laplacian for a weighted digraph with adjacency matrix $\mathbf{\Pi P}$. This last matrix has all row sums and column sums equal to each other: $\mathbf{\Pi P} \mathbf{1}=(\boldsymbol{\Pi P})^{T} \mathbf{1}=\boldsymbol{\pi}$, and hence the corresponding Laplacian is "Eulerian" [10, 11]. The matrix $\Pi^{1 / 2} \mathbf{P} \Pi^{-1 / 2}$ has a similar property: $\boldsymbol{\Pi}^{1 / 2} \mathbf{P} \boldsymbol{\Pi}^{-1 / 2} \sqrt{\boldsymbol{\pi}}=\left(\boldsymbol{\Pi}^{1 / 2} \mathbf{P} \boldsymbol{\Pi}^{-1 / 2}\right)^{T} \sqrt{\boldsymbol{\pi}}=\sqrt{\boldsymbol{\pi}}$. Here $\sqrt{\boldsymbol{\pi}}=\left[\sqrt{\pi_{1}}, \ldots, \sqrt{\pi_{n}}\right]^{T}$.

In this paper, we focus specifically on Laplacian matrices corresponding to strongly connected digraphs, specifically matrices $L$ such that are $(\mathrm{Pa})$ irreducible, $(\mathrm{Pb})$ have all positive diagonal entries and no positive off-diagonal entries, and (Pc) satisfy $L \mathbf{x}=0$ for some strictly positive vector $\mathbf{x}>0$. We call such a matrix an Eulerian Laplacian if $L^{T} \mathbf{x}=L \mathbf{x}=0$ for some strictly positive vector $\mathbf{x}>0$.

The main point of this section is to present the mapping between the pseudo-inverse computation for an Eulerian Laplacian and the computation of related ordinary inverses. In the following theorem we present two such mappings. Part (a) connects the pseudo-inverse of the Eulerian Laplacian matrix with the ordinary inverse of a symmetric rank-1 modification to that matrix. This is a special case of the general theory from [28]. The rank-1 modification is exactly in the direction corresponding to the nullspace of the original Laplacian. This construction is well known (see, e.g., [5, 14]). Part (b) shows how the ordinary inverse of the $(n-1) \times(n-1)$ principal submatrix of a Eulerian Laplacian can be obtained directly from the pseudoinverse of the entire matrix via small rank changes, and part (c) gives reverse mapping, from the ordinary inverse of the submatrix to the pseudo-inverse of the entire matrix. These connections will allow the use of off-the-shelf iterative methods for the ordinary inverse in order to obtain the desired pseudo-inverse. Part (b) is a special case of [5, Lemma 1]. We remark that for an Eulerian Laplacian, the Moore-Penrose pseudoinverse is the same as the group inverse [29, 33], and hence part (c) of Theorem 1 is a special case of [29, Thm 5.2].

Theorem 1. Let $C$ be an $n \times n$ non-singular matrix and suppose $A=C-\alpha \mathbf{u u}^{T}$ is singular with $A \mathbf{u}=$ $A^{T} \mathbf{u}=\mathbf{0}$. Partition $A$ and $\mathbf{u}$ as follows:

$$
A=\left[\begin{array}{ll}
A_{11} & \mathbf{a}_{12} \\
\mathbf{a}_{21}^{T} & a_{n n}
\end{array}\right], \quad \mathbf{u}=\left[\begin{array}{l}
\mathbf{u}_{1} \\
u_{n}
\end{array}\right] .
$$

Assume $\mathbf{u}^{T} \mathbf{u}=1$ and $u_{n}>0$. Then

(a) the left and right nullspaces of $A$ are $\operatorname{nullsp}(A)=\operatorname{nullsp}\left(A^{T}\right)=\operatorname{span}(\mathbf{u})$, and the Moore-Penrose pseudo-inverse of $A$ is given as:

$$
A^{\dagger} \stackrel{\text { def }}{=} B=C^{-1}-\mathbf{u u}^{T} / \alpha
$$

(b) $A_{11}^{-1}$ exists and can be written in terms of $A^{\dagger}=B$ :

$$
\begin{aligned}
A_{11}^{-1} & =\left[I_{n-1},-\mathbf{u}_{1} / u_{n}\right] B\left[\begin{array}{c}
I_{n-1} \\
-\mathbf{u}_{1}^{T} / u_{n}
\end{array}\right] \\
& =B_{11}-\frac{1}{u_{n}} \mathbf{u}_{1} \mathbf{b}_{21}^{T}-\frac{1}{u_{n}} \mathbf{b}_{12} \mathbf{u}_{1}^{T}+\frac{b_{n n}}{u_{n}^{2}} \mathbf{u}_{1} \mathbf{u}_{1}^{T},
\end{aligned}
$$

where we have partitioned $B$ as in (6). 
(c) We can write $A$ and $B=A^{\dagger}$ in terms of $A_{11}$ and $\mathbf{u}$ as follows

$$
\begin{aligned}
& A=\left[\begin{array}{cc}
A_{11} & -\frac{1}{u_{n}} A_{11} \mathbf{u}_{1} \\
-\frac{1}{u_{n}} \mathbf{u}_{1}^{T} A_{11} & \frac{1}{u_{n}^{2}} \mathbf{u}_{1}^{T} A_{11} \mathbf{u}_{1}
\end{array}\right]=\left[\begin{array}{c}
I_{n-1} \\
-\frac{1}{u_{n}} \mathbf{u}_{1}^{T}
\end{array}\right] A_{11}\left[I_{n-1},-\frac{1}{u_{n}} \mathbf{u}_{1}\right] ; \\
& B=\left[\begin{array}{ll}
B_{11} & \mathbf{b}_{12} \\
\mathbf{b}_{21}^{T} & b_{n n}
\end{array}\right]=\left[\begin{array}{c}
I_{n-1}-\mathbf{u}_{1} \mathbf{u}_{1}^{T} \\
-u_{n} \cdot \mathbf{u}_{1}^{T}
\end{array}\right] A_{11}^{-1}\left[I_{n-1}-\mathbf{u}_{1} \mathbf{u}_{1}^{T},-u_{n} \cdot \mathbf{u}_{1}\right]
\end{aligned}
$$

where the individual blocks are

$$
\begin{aligned}
& B_{11}=A_{11}^{-1}-\mathbf{u}_{1} \mathbf{t}^{T}-\mathbf{w} \mathbf{u}_{1}^{T}+\left(\mathbf{u}_{1}^{T} \mathbf{w}\right) \cdot \mathbf{u}_{1} \mathbf{u}_{1}^{T} \\
& \mathbf{b}_{12}=u_{n}\left(\mathbf{u}_{1}^{T} \mathbf{w}\right) \cdot \mathbf{u}_{1}-u_{n} \cdot \mathbf{w} \\
& \mathbf{b}_{21}^{T}=u_{n}\left(\mathbf{u}_{1}^{T} \mathbf{w}\right) \cdot \mathbf{u}_{1}^{T}-u_{n} \cdot \mathbf{t}^{T} \\
& b_{n n}=u_{n}^{2}\left(\mathbf{u}_{1}^{T} \mathbf{w}\right) \\
& \text { where } \mathbf{w}=A_{11}^{-1} \mathbf{u}_{1}, \quad \mathbf{t}^{T}=\mathbf{u}_{1}^{T} A_{11}^{-1}
\end{aligned}
$$

\section{Proof.}

(a) A simple calculation yields $A B=B A=I_{n}-\mathbf{u u}^{T}$, and a further simple calculation yields $A B A=A$ and $B A B=B$. Hence $B$ satisfies the conditions to be the Moore-Penrose pseudo inverse.

(b) $A \mathbf{u}=\mathbf{0}$ and $\mathbf{u}^{T} A=\mathbf{0}^{T}$ imply

$$
\begin{array}{ll}
A_{11} \mathbf{u}_{1}=-\mathbf{a}_{12} u_{n}, & \mathbf{u}_{1}^{T} A_{11}=-\mathbf{a}_{21}^{T} u_{n} \\
\mathbf{a}_{21}^{T} \mathbf{u}_{1}=-a_{n n} u_{n}, & \mathbf{u}_{1}^{T} \mathbf{a}_{12}=-a_{n n} u_{n}
\end{array}
$$

Likewise, $B \mathbf{u}=\mathbf{0}$ and $\mathbf{u}^{T} B=\mathbf{0}^{T}$ imply

$$
\begin{array}{ll}
B_{11} \mathbf{u}_{1}=-\mathbf{b}_{12} u_{n}, & \mathbf{u}_{1}^{T} B_{11}=-\mathbf{b}_{21}^{T} u_{n} \\
\mathbf{b}_{21}^{T} \mathbf{u}_{1}=-b_{n n} u_{n}, & \mathbf{u}_{1}^{T} \mathbf{b}_{12}=-b_{n n} u_{n}
\end{array}
$$

These yield the equivalence for the two formulas for $A_{11}^{-1}$ in (7) and the formulas for $A$ in $(8)$. To verify (7) is indeed the inverse of $A_{11}$, we multiply (7) by $A_{11}$ to obtain the identity:

$$
\begin{aligned}
& {\left[I_{n-1}, \quad-\mathbf{u}_{1} / u_{n}\right] B\left[\begin{array}{c}
I_{n-1} \\
-\mathbf{u}_{1}^{T} / u_{n}
\end{array}\right] \cdot A_{11}} \\
& =\left[I_{n-1},-\mathbf{u}_{1} / u_{n}\right] B\left[\begin{array}{c}
A_{11} \\
-\mathbf{a}_{21}^{T}
\end{array}\right] \\
& =\left[I_{n-1},-\mathbf{u}_{1} / u_{n}\right]\left[\begin{array}{c}
I_{n-1}-\mathbf{u}_{1} \mathbf{u}^{T} \\
-u_{n} \mathbf{u}_{1}^{T}
\end{array}\right]=I_{n-1}
\end{aligned}
$$

(c) Using the second formulas for $A, B$ in (8), calculations similar to the proof of (b) yield $A B=B A=$ $I_{n}-\mathbf{u u}^{T}$ and then $A B A=A, B A B=B$.

\section{Algorithm for Eulerian Laplacians.}

We study the computation of the pseudo-inverses of $\mathbf{L}^{\mathrm{d}}$ and $\mathbf{L}^{\mathrm{r}}$. Using Theorem 1 , we can write these as follows:

$$
\begin{aligned}
& {[\mathrm{a}] \mathbf{M}^{\mathrm{d}} \stackrel{\text { def }}{=}\left(\mathbf{L}^{\mathrm{d}}\right)^{\dagger}=\left(\mathbf{L}^{\mathrm{d}}+\sqrt{\boldsymbol{\pi}} \sqrt{\boldsymbol{\pi}}^{T}\right)^{-1}-\sqrt{\boldsymbol{\pi}} \sqrt{\boldsymbol{\pi}}^{T}} \\
& {[\mathrm{~b}] \quad \mathbf{M}^{\mathrm{r}} \stackrel{\text { def }}{=}\left(\mathbf{L}^{\mathrm{r}}\right)^{\dagger}=\left(\mathbf{L}^{\mathrm{r}}+\alpha \frac{\mathbf{1 1}^{T}}{n}\right)^{-1}-\frac{1}{\alpha} \frac{\mathbf{1 1}^{T}}{n},}
\end{aligned}
$$


for some arbitrary $\alpha \neq 0$ (we use $\alpha=1$ below, but include it here to show a slightly more general formula). Theorem 1 applies here because both $\mathbf{L}^{\mathrm{r}}$ and $\mathbf{L}^{\mathrm{d}}$ are Eulerian.

The overall algorithm begins with a computation of the stationary probabilities. These probabilities are used to scale non-Eulerian Laplacians to an Eulerian scaling. The final step is to solve for the pseudo-inverse of the Eulerian Laplacian by applying an iterative method to (9). The detailed steps are given in Algorithm 1.

\section{Algorithm 1.}

Input: $\mathbf{P}=$ probability transition matrix for a random walk over the graph and an index set $\mathcal{J} \subset\{1, \ldots, n\}$. Output: Stationary probabilities $\boldsymbol{\pi}$ and the columns indexed by $\mathcal{J}$ of either [a] pseudo-inverse $\mathbf{M}^{\mathrm{d}}=$ $\left(\mathbf{L}^{\mathrm{d}}\right)^{\dagger}=\left(\mathbf{I}-\boldsymbol{\Pi}^{1 / 2} \mathbf{P} \boldsymbol{\Pi}^{-1 / 2}\right)^{\dagger}$, or [b] pseudo-inverse $\mathbf{M}^{\mathrm{r}}=\left(\mathbf{L}^{\mathrm{r}}\right)^{\dagger}=(\boldsymbol{\Pi}-\boldsymbol{\Pi} \mathbf{P})^{\dagger}$. Note: items marked [a] are needed only for $\mathbf{M}^{\mathrm{d}}$ while items marked [b] are needed only for $\mathbf{M}^{\mathrm{r}}$.

1. Compute $\pi$, the vector of stationary probabilities:

Use the modified subspace iteration method with $\ell$ starting vectors [37] on $\mathbf{P}^{T}$ to compute eigenvector corresponding to the eigenvalue $\lambda=1$. Here $\ell$ is larger than the period of the graph.

2. Set $[\mathrm{a}] \mathbf{L}^{\mathrm{d}}=\mathbf{I}-\boldsymbol{\Pi}^{1 / 2} \mathbf{P} \boldsymbol{\Pi}^{-1 / 2}$, where $\boldsymbol{\Pi}^{1 / 2}=\operatorname{Diag}(\sqrt{\boldsymbol{\pi}})$, or

[b] $\mathbf{L}^{\mathrm{r}}=\boldsymbol{\Pi}-\boldsymbol{\Pi} \mathbf{P}$, where $\boldsymbol{\Pi}=\operatorname{Diag}(\boldsymbol{\pi})$.

3. Compute the selected columns indexed by $\mathcal{J}$ of pseudo-inverse of the Eulerian Laplacian using (9): either [a] $\mathbf{M}^{\mathrm{d}}=\left(\mathbf{L}^{\mathrm{d}}\right)^{\dagger}$ or [b] $\mathbf{M}^{\mathrm{r}}=\left(\mathbf{L}^{\mathrm{r}}\right)^{\dagger}$ column-by-column as follows:

For $j \in \mathcal{J}$ :

(i) Solve the following linear systems using restarted GMRES $(\ell)$ for $\mathrm{x}^{\mathrm{d}}$ and $\mathrm{x}^{\mathrm{r}}$ :

$$
\begin{aligned}
& \text { [a] }\left(\mathbf{L}^{\mathrm{d}}+\sqrt{\boldsymbol{\pi}} \sqrt{\boldsymbol{\pi}}^{T}\right) \mathbf{x}^{\mathrm{d}}=\mathbf{e}_{j}, \text { or } \\
& \text { [b] }\left(\mathbf{L}^{\mathrm{r}}+\mathbf{1 1}^{T} / n\right) \mathbf{x}^{\mathrm{r}}=\mathbf{e}_{j} .
\end{aligned}
$$

(ii) Fill in the $j$-th column of pseudo-inverse:

$$
\begin{aligned}
& \text { [a] } \mathbf{M}_{:, j}^{\mathrm{d}}=\mathbf{x}^{\mathrm{d}}-\sqrt{\pi_{j}} \cdot \sqrt{\boldsymbol{\pi}}, \text { or } \\
& \text { [b] } \mathbf{M}_{:, j}^{\mathrm{r}}=\mathbf{x}^{\mathrm{r}}-1 / n \mathbf{1},
\end{aligned}
$$

\section{Complexity of Algorithm 1: Convergence.}

The two most expensive steps in Algorithm 1 are steps 1 and 3 $(i)$, both involving an iterative method. Their cost is a product of the cost per iteration times the number of iterations. Of the remaining steps, step 2 involves diagonal scaling which costs only $O(m)$ operations, since only the nonzero elements must be computed. Step 3 (ii) costs $O(n)$ for each column or $O(n|\mathcal{J}|)$ altogether. If the entire pseudo-inverse were to be computed, it could cost $O\left(n^{2}\right)$ overall, i.e., constant time per matrix entry.

In step 1 the modified subspace iteration (see Algorithm 3] in the Appendix) [37] computes the Schur decomposition of a small $\ell \times \ell$ matrix which is the orthogonal projection of the original matrix $\mathbf{P}^{T}$ onto an $\ell$ dimensional subspace. If $\lambda_{1}, \lambda_{2}, \ldots, \lambda_{n}$ are the eigenvalues of $\mathbf{P}$ with $1=\lambda_{1} \geq\left|\lambda_{2}\right| \geq\left|\lambda_{\ell}\right|>\left|\lambda_{\ell+1}\right| \geq$ $\cdots \geq\left|\lambda_{n}\right|$, and $\lambda_{1}=1$ is a simple eigenvalue, then Stewart [37] showed that the leading eigenvector (corresponding to $\lambda_{1}=1$ ) converges as:

$$
\frac{\left\|\mathbf{P}^{T} \mathbf{x}^{[k]}-\mathbf{x}^{[k]}\right\|}{\left\|\mathbf{P}^{T} \mathbf{x}^{[0]}-\mathbf{x}^{[0]}\right\|} \leq O\left(\left|\lambda_{\ell+1}\right|^{k}\right)
$$

where $\mathbf{x}^{[k]}$ denotes the approximation to the eigenvector corresponding to $\lambda_{1}=1$ at the $k$-iteration. In the following we use $c_{1}, c_{2}, \ldots$ to represent small positive constants in the costs bounds, all of which are less than 10. If the random walk is aperiodic, then we are guaranteed that $1>\left|\lambda_{2}\right| \geq \cdots \geq\left|\lambda_{\ell}\right| \geq \cdots$. If the 
random walk is periodic with period per, it suffices to have $\ell>$ per in order to guarantee that $\lambda_{\ell+1}<\lambda_{1}=1$. To obtain an error at most tol requires at least $c^{\mathrm{s}}$ iterations with

$$
c^{\mathrm{s}} \geq \frac{\mid \log (\text { tol })|+| \log \left(\left\|\mathbf{P}^{T} \mathbf{x}^{[0]}-\mathbf{x}^{[0]}\right\|\right) \mid}{\left|\log \left(\lambda_{\ell+1}\right)\right|}
$$

As written in Algorithm 3 in the Appendix, the cost per iteration is $\operatorname{cost}_{1}^{\text {subspace }}=O\left(\operatorname{cost}_{M V} \cdot(\ell+1)+\right.$ $\left.\left(n \ell^{2}\right)+\left(\ell^{3}\right)\right)$, where $\operatorname{cost}_{M_{v}}$ is the cost of one matrix-vector product, proportional to the number of nonzero entries in the matrix, $\mathrm{nnz}($ matrix $)$. The storage required is space subspace $^{2} O(\#$ edges $\left.+n)+(n \ell)+\ell^{2}\right) \mathrm{We}$ remark that for undirected graphs, this eigenvector is a multiple of the vector of degrees, so this step would be essentially free.

We remark that there are many choices of algorithms to compute this eigenvector, similar to the many choices to compute the pagerank vector, many of which can be faster [16]. If the dimension is small enough, one can use solve for the eigenvector directly by finding $\mathbf{v}^{T}$ satisfying the homogeneous linear system

$$
\left[\mathbf{v}^{T}, 1\right]\left[\begin{array}{c}
\mathbf{P}_{11} \\
\mathbf{p}_{21}^{T}
\end{array}\right]=\mathbf{v}^{T},
$$

where $\mathbf{P}_{11}$ is the upper left $(n-1) \times(n-1)$ block of $\mathbf{P}$, but with $O\left(n^{3}\right)$ cost with $O\left(n^{2}\right)$ space using ordinary Gaussian elimination. Modified Subspace Iteration is an effective algorithm which enjoys a simple bound on its convergence rate and fixed cost per iteration and little additional space beyond that of the input matrix, leading to a simple complexity bound.

The other costly step is step 3 (i) to compute a column of the inverse $\mathbf{M}^{\mathrm{d}}$. This line is called $|\mathcal{J}|$ times, each time solving a linear system to obtain one column of the inverse. To solve the linear system, we use an iterative method with a fixed bound on the cost per iteration and a convergence guarantee yielding a bound on the number of iterations depending on the accuracy desired but not on the dimensions of the graph. For this purpose we use GMRES $(\ell)$, i.e., restarted GMRES where $\ell$ is the number of inner steps between restarts, because it not only enjoys these theoretical properties, but has also been observed to be a very effective solver in practice [34, 35]. The cost of one outer iteration of restarted GMRES is (details in the Appendix) $\operatorname{cost}_{1}^{\mathrm{GMRES}}=O\left(\ell \cdot \operatorname{cost}_{M v}+n \ell^{2}+\ell^{2}\right)$. In order to complete the complexity bound for this step, we must show that restarted GMRES converges at a guaranteed rate. In order to do that, we show that the symmetric part of the modified Laplacian matrices in question are positive definite, in the following lemma.

Lemma 2. If $\mathbf{P}$ is the probability transition matrix for a strongly connected directed graph, and $\boldsymbol{\pi}>0$ is the vector of stationary probabilities, then the following two matrices

$$
\begin{array}{lll}
S\left(\mathbf{L}^{\mathrm{d}}\right)+\sqrt{\boldsymbol{\pi}} \sqrt{\boldsymbol{\pi}}^{T} & \stackrel{\text { def }}{=} & \left(\mathbf{L}^{\mathrm{d}}+\left(\mathbf{L}^{\mathrm{d}}\right)^{T}\right) / 2+\sqrt{\boldsymbol{\pi}} \sqrt{\boldsymbol{\pi}}^{T} \\
S\left(\mathbf{L}^{\mathrm{r}}\right)+\mathbf{1 1}^{T} & \stackrel{\text { def }}{=} & \left(\mathbf{L}^{\mathrm{r}}+\left(\mathbf{L}^{\mathrm{r}}\right)^{T}\right) / 2+\mathbf{1 1}^{T}
\end{array}
$$

are symmetric positive definite.

Proof (sketch). We show the symmetric part of the non-symmetric Laplacian is the Laplacian for a weighted undirected graph and hence is an M-matrix [3] which is positive semidefinite. Consider the weighted undirected graph with adjacency matrix $\widetilde{\mathbf{A}}=\left(\boldsymbol{\Pi} \mathbf{P}+\mathbf{P}^{T} \boldsymbol{\Pi}\right) / 2$. This is a weighted undirected graph with the same nodes as the original graph and an edge whereever the original graph has an edge in either direction. The vector of stationary probabilities for this graph is $\pi$, proportional to the weighted degrees of the nodes in the new graph. The associated unnormalized Laplacian is $1 / 2\left(\mathbf{L}^{\mathrm{r}}+\left(\mathbf{L}^{\mathrm{r}}\right)^{T}\right)$, which is therefore symmetric positive semi-definite with nullspace equal to $\operatorname{span}(\mathbf{1})$ [9]. The associated diagonally scaled 
Laplacian is $1 / 2\left(\mathbf{L}^{\mathrm{d}}+\left(\mathbf{L}^{\mathrm{d}}\right)^{T}\right)=1 / 2 \boldsymbol{\Pi}^{-1 / 2}\left(\mathbf{L}^{\mathrm{r}}+\left(\mathbf{L}^{\mathrm{r}}\right)^{T}\right) \boldsymbol{\Pi}^{-1 / 2}$, which is therefore also symmetric positive semi-definite with nullspace equal to $\operatorname{span}(\sqrt{\pi})$. The probability transition matrix for the new graph is $\widetilde{\mathbf{P}}=\boldsymbol{\Pi}^{-1} \widetilde{\mathbf{A}}=\left(\mathbf{P}+\boldsymbol{\Pi}^{-1} \mathbf{P}^{T} \boldsymbol{\Pi}\right) / 2$. Adding a symmetric rank-1 matrix $\left(\mathbf{1 1}^{T}\right.$ or $\sqrt{\boldsymbol{\pi}} \sqrt{\boldsymbol{\pi}}^{T}$, respectively $)$ makes the respective Laplacian matrices non-singular, moving the 0 eigenvalue to a positive number without moving the remaining eigenvalues.

We can now notice that the Laplacian matrices $S\left(\mathbf{L}^{\mathrm{r}}\right)+\sqrt{\boldsymbol{\pi}} \sqrt{\boldsymbol{\pi}}^{T}, S\left(\mathbf{L}^{\mathrm{d}}\right)+\mathbf{1 1}^{T}$ have just the right scaling to belong to a class of matrices for which GMRES (or any similar Krylov space minimum residual method) has a guaranteed convergence rate. We have the following theorem that is an immediate consequence of Theorem 5 in the Appendix.

Corollary 3. Let $A$ be a real matrix such that $S(A)=\left(A+A^{T}\right) / 2$ is symmetric positive definite and let $\lambda_{\min }[S(A)]>0$ denote the smallest eigenvalue for $S(A)$. The residual $\mathbf{r}_{k}$ obtained by restarted GMRES [34] (restarting after $\ell$ inner steps) after $k$ outer steps satisfies

$$
\frac{\left\|\mathbf{r}_{k}\right\|_{2}}{\left\|\mathbf{r}_{0}\right\|_{2}} \leq\left(1-\frac{\left(\lambda_{\min }[S(A)]\right)^{2}}{\|A\|_{2}^{2}}\right)^{k \ell / 2}
$$

The cost of one outer step of restarted GMRES is $\cos _{1}^{\mathrm{GMRES}} \ell \cdot \operatorname{cost}_{M_{v}}+c_{7}\left(n \ell^{2}+\ell^{2}\right)$.

Proof. According to Theorem 5 the residual after $\ell$ steps of ordinary GMRES satisfies

$$
\frac{\left\|\mathbf{r}_{\ell}\right\|_{2}}{\left\|\mathbf{r}_{0}\right\|_{2}} \leq\left(1-\frac{\left(\lambda_{\min }[S(A)]\right)^{2}}{\|A\|_{2}^{2}}\right)^{\ell / 2}
$$

Each time GMRES is restarted after $\ell$ steps, the residual is reduced by the factor in equ. (12). After $k$ such repeats, the residual has been reduced by a factor of at least (11). The cost estimate is based on an analysis of Algorithm 4, detailed in [34], as sketched in the Appendix.

In summary, the total cost to find the vector of stationary probabilities is

$$
\begin{aligned}
\operatorname{cost}^{\text {subspace }} & =c^{\mathrm{s}}\left((\ell+1) \cdot \operatorname{cost}_{M_{V}}+c_{8}\left(2 n \ell^{2}+\ell^{3}\right)\right) \\
& =\frac{\left\lfloor\log (t o l)|+| \log \left\|\mathbf{r}_{0}\right\| \mid\right.}{|\log \rho|}\left((\ell+1) \cdot \operatorname{cost}_{M_{V}}+c_{8}\left(n \ell^{2}+\ell^{3}\right)\right),
\end{aligned}
$$

where $\rho=\lambda_{\ell+1}(\mathbf{P})$. The cost to find each column of the pseudo-inverse with a residual error of tol, given the vector of stationary probabilities is

$$
\begin{aligned}
\operatorname{cost}^{\mathrm{GMRES}} & =c^{\mathrm{g}}\left(\ell \cdot \operatorname{cost}_{M_{V}}+c_{7}\left(n \ell^{2}+\ell^{2}\right)\right) \\
& =\frac{\left\lfloor\log (\mathrm{tol})|+| \log \left\|\mathbf{r}_{0}\right\|||\right.}{|\log \sigma|}\left(\ell \cdot \operatorname{cost}_{M_{V}}+c_{7}\left(n \ell^{2}+\ell^{2}\right)\right)
\end{aligned}
$$

where $\sigma=\left(1-\frac{\left(\lambda_{\min }[S(A)]\right)^{2}}{\|A\|_{2}^{2}}\right)^{\ell / 2}$ and $c^{\mathrm{g}}=\frac{|\log (\mathrm{tol})|+\left|\log \left\|\mathbf{r}_{0}\right\|\right|}{|\log \sigma|}$. Hence the total cost to obtain one column of the pseudo-inverse of an Eulerian Laplacian equal to:

$$
\cos ^{\mathrm{one}}=\left(c^{\mathrm{s}}+c^{\mathrm{g}}\right)\left(\ell \cdot \operatorname{cost}_{M_{V}}+c_{9}\left(n+n \ell^{2}+\ell^{3}\right)\right),
$$

where cost $_{M v}=O(m)$ is proportional the number of edges in the graph. The space required is space $=$ $O(\#$ edges $\left.+n)+(n \ell)+\ell^{2}\right)$, where $\ell$ is the number of inner iterations of GMRES or the number of vectors used in the subspace iteration (whichever is bigger). We remark that in order to obtain the hitting time $h(i, k)$ for a given node $k$, or to obtain the trust or influence measure [17, 27] for a given node $j$, only one column of the pseudo-inverse is required at a cost shown in (13). Obtaining the entire pseudo-inverse, in 
cases where that would be required, requires the computation of the stationary probabilities only once, so the total cost would be bounded by:

$$
\operatorname{cost}^{\mathrm{total}} \leq\left(c^{\mathrm{s}}+n c^{\mathrm{g}}\right)\left(\ell \cdot \cos _{M_{V}}+c_{9}\left(n+n \ell^{2}+\ell^{3}\right)\right) .
$$

\section{General Pseudo-Inverses.}

Here we show how to apply the previous to obtain the inverses or pseudo-inverses of general Laplacian matrices derived from strongly connected directed graphs. The approach is to apply a row/column diagonal scaling to the non-Eulerian Laplacian to obtain a related Eulerian matrix (similar to [11]), compute the pseudo-inverse using the previous methods, and unwind the diagonal scaling. This can be applied to any Laplacian $\widetilde{\mathbf{L}}$ that has all the following properties:

(Pa) $\widetilde{\mathbf{L}}$ is irreducible,

$(\mathrm{Pb})$ all the diagonal entries are strictly positive and all the off-diagonal entries are non-positive,

(Pc) there is a strictly positive vector $\mathrm{x}$ so that $\widetilde{\mathbf{L}} \mathbf{x}=0$.

Matrices satisfying property $(\mathrm{Pb})$ are Z-matrices [3]. Alternatively, we can start with an $(n-1) \times(n-1)$ matrix $\widetilde{\mathbf{L}}_{11}$ which shares properties $(\mathrm{Pa})$ and $(\mathrm{Pb})$, but has the property

(Pc') there is a strictly positive vector $\mathbf{w}$ so that $\widetilde{\mathbf{L}}_{11} \mathbf{w}$ is strictly positive.

Then we use Theorem 4 (c) below to embed $\widetilde{\mathbf{L}}_{11}$ inside an $n \times n$ matrix $\widetilde{\mathbf{L}}$ enjoying properties $(\mathrm{Pa}),(\mathrm{Pb}),(\mathrm{Pc})$ and hence apply the same procedures. Matrices satisfying $(\mathrm{Pa}),(\mathrm{Pb}),(\mathrm{Pc})$ are non-singular M-matrices and include matrices that are strictly row-diagonally dominant with non-positive off-diagonal entries [3]. There are many other ways to characterize M-matrices (see [3]).

The pseudo-inverse of a diagonally scaled matrix is not the diagonally scaled pseudo-inverse of the original, but the ordinary inverse of a diagonally scaled matrix is the diagonally scaled ordinary inverse of the original. Hence one can apply the diagonal scaling to the leading principal submatrix of a Laplacian to map the problem to the Eulerian scaling. The following theorem provides a way to map from a matrix pseudo-inverse to the ordinary inverse of its principal submatrix and vice versa, using only fast rank-one updates. Alternatively, one can use $\Pi$ as a preconditioner on the unscaled Laplacian.

Theorem 4. Let $C$ be an $n \times n$ non-singular matrix and suppose $A=C-\alpha \mathbf{u v}^{T}$ is singular with $A \mathbf{u}=$ $A^{T} \mathbf{v}=\mathbf{0}, \mathbf{v}^{T} \mathbf{u}=1$ and $u_{n}>0, v_{n}>0$. Then

(a) the left and right nullspaces of $A$ are $\operatorname{nullsp}(A)=\operatorname{span}(\mathbf{u})$, and $\operatorname{nullsp}\left(A^{T}\right)=\operatorname{span}(\mathbf{v})$. The MoorePenrose pseudo-inverse of $A$ is given as:

$$
\begin{aligned}
A^{\dagger} & =B \stackrel{\text { def }}{=}\left(I_{n}-\frac{1}{\mathbf{u}^{T} \mathbf{u}} \mathbf{u} \mathbf{u}^{T}\right) C^{-1}\left(I_{n}-\frac{1}{\mathbf{v}^{T} \mathbf{v}} \mathbf{v} \mathbf{v}^{T}\right) \\
& =C^{-1}-\frac{1}{\mathbf{u}^{T} \mathbf{u}} \mathbf{u} \mathbf{y}^{T}-\frac{1}{\mathbf{v}^{T} \mathbf{v}} \mathbf{x} \mathbf{v}^{T}+\frac{\mathbf{u}^{T} \mathbf{x}}{\left(\mathbf{u}^{T} \mathbf{u}\right)\left(\mathbf{v}^{T} \mathbf{v}\right)} \mathbf{u} \mathbf{v}^{T},
\end{aligned}
$$

where $\mathbf{y}^{T}=\mathbf{u}^{T} C^{-1}, \quad \mathbf{x}=C^{-1} \mathbf{v}$.

(b) $A_{11}^{-1}$ exists and can be written in terms of $A^{\dagger}=B, \mathbf{u}, \mathbf{v}$ :

$$
\begin{aligned}
A_{11}^{-1} & =\left(I_{n-1}+\frac{1}{u_{n}^{2}} \mathbf{u}_{1} \mathbf{u}_{1}^{T}\right) B_{11}\left(I_{n-1}+\frac{1}{v_{n}^{2}} \mathbf{v}_{1} \mathbf{v}_{1}^{T}\right) \\
& =\left[I_{n-1},-\mathbf{u}_{1} / u_{n}\right] B\left[\begin{array}{c}
I_{n-1} \\
-\mathbf{v}_{1}^{T} / v_{n}
\end{array}\right] \\
& =B_{11}-\frac{1}{u_{n}} \mathbf{u}_{1} \mathbf{b}_{21}^{T}-\frac{1}{v_{n}} \mathbf{b}_{12} \mathbf{v}_{1}^{T}+\frac{b_{n n}}{u_{n} v_{n}} \mathbf{u}_{1} \mathbf{v}_{1}^{T}
\end{aligned}
$$


where we have partitioned $B$ as above.

(c) We can write $A$ and $B=A^{\dagger}$ in terms of $A_{11}, \mathbf{u}, \mathbf{v}$ as follows

$$
\begin{aligned}
& A=\left[\begin{array}{cc}
A_{11} & -\frac{1}{u_{n}} A_{11} \mathbf{u}_{1} \\
-\frac{1}{v_{n}} \mathbf{v}_{1}^{T} A_{11} & \frac{\mathbf{v}_{1}^{f} A_{11} \mathbf{u}_{1}}{u_{n} v_{n}}
\end{array}\right]=\left[\begin{array}{c}
I_{n-1} \\
-\frac{1}{v_{n}} \mathbf{v}_{1}^{T}
\end{array}\right] A_{11}\left[I_{n-1},-\frac{1}{u_{n}} \mathbf{u}_{1}\right] \\
& B=\left[\begin{array}{ll}
B_{11} & \mathbf{b}_{12} \\
\mathbf{b}_{21}^{T} & b_{n n}
\end{array}\right]=\left[\begin{array}{c}
I_{n-1}-\frac{\mathbf{u}_{1} \mathbf{u}_{1}^{T}}{\mathbf{u}^{T} \mathbf{u}} \\
-\frac{u_{n}}{\mathbf{u}^{T} \mathbf{u}} \cdot \mathbf{u}_{1}^{T}
\end{array}\right] A_{11}^{-1}\left[I_{n-1}-\frac{\mathbf{v}_{1} \mathbf{v}_{1}^{T}}{\mathbf{v}^{T} \mathbf{v}},-\frac{v_{n}}{\mathbf{v}^{T} \mathbf{v}} \cdot \mathbf{v}_{1}\right]
\end{aligned}
$$

where the individual blocks of $B$ are

$$
\begin{aligned}
& \text { (a) } B_{11}=A_{11}^{-1}-\mathbf{u}_{1} \mathbf{t}^{T}-\mathbf{w} \mathbf{v}_{1}^{T}+\frac{\mathbf{u}_{1}^{T} \mathbf{w}}{\mathbf{u}^{T} \mathbf{u}} \cdot \mathbf{u}_{1} \mathbf{v}_{1}^{T} \\
& \text { (b) } \mathbf{b}_{12}=v_{n} \frac{\mathbf{u}_{1}^{T} \mathbf{w}}{\mathbf{u}^{T} \mathbf{u}} \cdot \mathbf{u}_{1}-v_{n} \mathbf{w} \\
& \text { (c) } \mathbf{b}_{21}^{T}=u_{n} \frac{\mathbf{u}_{1}^{T} \mathbf{w}}{\mathbf{u}^{T} \mathbf{u}} \cdot \mathbf{v}_{1}^{T}-u_{n} \mathbf{t}^{T} \\
& \text { (d) } b_{n n}=u_{n} v_{n} \frac{\mathbf{u}_{1}^{T} \mathbf{w}}{\mathbf{u}^{T} \mathbf{u}} \\
& \text { where } \mathbf{w}=\frac{1}{\mathbf{v}^{T} \mathbf{v}} A_{11}^{-1} \mathbf{v}_{1}, \quad \mathbf{t}^{T}=\frac{1}{\mathbf{u}^{T} \mathbf{u}} \mathbf{u}_{1}^{T} A_{11}^{-1}
\end{aligned}
$$

Part (a) is a special case of general theory of [28], and part (b) appears in [5]. Part (c) give a formula for the Moore-Penrose pseudo-inverse that is similar to the formula in [29] for the so-called group inverse, but these two inverses agree only when the left and right nullspaces match [33].

Proof. The proof follows the same lines as that of Theorem 11 after noting that $A B=I-\frac{\mathbf{v v}^{T}}{\mathbf{v}^{T} \mathbf{v}}$, and $B A=I-\frac{\mathbf{u u}^{T}}{\mathbf{u}^{T} \mathbf{u}}$.

Using this theorem, we briefly outline a feasible procedure to obtain the [pseudo]-inverse for an admissible Laplacian matrix consisting of a sequence of diagonal scalings and the Eulerian Laplacian Algorithm 1.

Suppose we have a matrix $\widetilde{\mathbf{L}}$ satisfying properties $(\mathrm{Pa}),(\mathrm{Pb}),(\mathrm{Pc})$, together with a strictly positive vector $\mathbf{x}$ such that $0=\widetilde{\mathbf{L}} \mathbf{x}$. For example, an adjacency matrix $\widetilde{\mathbf{A}}$ for a strongly connected digraph, with associated vector of out-degrees $\tilde{\mathbf{d}}$, leads to the unnormalized Laplacian, $\widetilde{\mathbf{L}}=\operatorname{Diag}(\tilde{\mathbf{d}})-\widetilde{\mathbf{A}}$, with $\mathbf{x}=$ 1. Define $\widehat{\mathbf{L}} \stackrel{\text { def }}{=}(\widehat{\mathbf{D}}-\widehat{\mathbf{A}})=\widetilde{\mathbf{L}} \cdot \operatorname{Diag}(\mathbf{x})$, where $\widehat{\mathbf{D}}$ is the diagonal part of $\widehat{\mathbf{L}}$, and $-\widehat{\mathbf{A}}$ has the rest. This matrix has the property that $\widehat{\mathbf{L}} \mathbf{1}=0$ while sharing the same left annihilating vector with $\widetilde{\mathbf{L}}$. In this case, $\widehat{\mathbf{P}}=\widehat{\mathbf{D}}^{-1} \widehat{\mathbf{A}}$ is the probability transition matrix for a random walk over this digraph, with strictly positive stationary probabilities $\boldsymbol{\pi}$. We can then apply the diagonal scaling to $\widehat{\mathbf{L}}$ to obtain the Eulerian Laplacian $\mathbf{L}^{\mathrm{d}}=\boldsymbol{\Pi}^{1 / 2}\left(\widehat{\mathbf{D}}^{-1} \widehat{\mathbf{L}}\right) \boldsymbol{\Pi}^{-1 / 2}$, and then use Algorithm 1 to compute its pseudo-inverse. The following algorithm uses this preprocessing to obtain the pseudo-inverse of the original matrix $\widetilde{\mathbf{L}}$.

\section{Algorithm 2.}

Input: $\widetilde{\mathbf{L}}$ satisfying $(\mathrm{Pa})-(\mathrm{Pc})$ and a strictly positive vector $\mathbf{x}$ such that $\widetilde{\mathbf{L}} \mathbf{x}=0$.

Output; Pseudo-inverse of $\widetilde{\mathbf{L}}$.

1. Form $\widehat{\mathbf{L}} \stackrel{\text { def }}{=} \widetilde{\mathbf{L}} \cdot \operatorname{Diag}(\mathbf{x})=(\widehat{\mathbf{D}}-\widehat{\mathbf{A}})$, where $\widehat{\mathbf{D}}=\widetilde{\mathbf{D}} \cdot \operatorname{Diag}(\mathbf{x})$ is the diagonal part of $\widehat{\mathbf{L}}$, and $\mathbf{A}$ has the rest.

2. Form the probability transition matrix $\widehat{\mathbf{P}}^{T}=\left(\widehat{\mathbf{D}}^{-1} \widehat{\mathbf{A}}\right)^{T}$. 
3. (*) Use Algorithm 1 to compute the vector of stationary probabilities $\pi$ and the pseudo-inverse $\left(\mathbf{L}^{\mathrm{d}}\right)^{\dagger}$ of $\mathbf{L}^{\mathrm{d}} \stackrel{\text { def }}{=} \boldsymbol{\Pi}^{1 / 2}\left(I-\widehat{\mathbf{D}}^{-1} \widehat{\mathbf{A}}\right) \boldsymbol{\Pi}^{-1 / 2}=\boldsymbol{\Pi}^{1 / 2}\left(\widehat{\mathbf{D}}^{-1} \widehat{\mathbf{L}}\right) \boldsymbol{\Pi}^{-1 / 2}$.

4. (*) Use Theorem 1 b) to obtain the $(n-1) \times(n-1)$ matrix $\left(\mathbf{L}_{11}^{\mathrm{d}}\right)^{-1}$ from $\left(\mathbf{L}^{\mathrm{d}}\right)^{\dagger}$. Here $\mathbf{u}=\sqrt{\boldsymbol{\pi}}$.

5. (*) Form the $(n-1) \times(n-1)$ matrix $\left(\widetilde{\mathbf{L}}_{11}\right)^{-1}=\left(\operatorname{Diag}\left(\mathbf{x}_{1: n-1}\right)\right) \mathbf{\Pi}_{1}^{-1 / 2}\left(\mathbf{L}_{11}^{\mathrm{d}}\right)^{-1} \mathbf{\Pi}_{1}^{1 / 2} \widehat{\mathbf{D}}_{1}^{-1}$.

6. (*) Use Theorem 4(c) to obtain $\widetilde{\mathbf{L}}^{\dagger}$ from $\left(\widetilde{\mathbf{L}}_{11}\right)^{-1}$ : Use (14) with $A_{11}^{-1}=\left(\widetilde{\mathbf{L}}_{11}\right)^{-1}, \mathbf{u}=\mathbf{x}$, and $\mathbf{v}=\widetilde{\mathbf{D}}^{-1} \boldsymbol{\pi}$, i.e., $v_{i}=\pi_{i} /\left(\tilde{d}_{i}\right), i=1, \ldots, n$. The vectors $\mathbf{u}, \mathbf{v}$ are the right and left annihilating vectors for the original Laplacian $\widetilde{\mathbf{L}}$.

In the steps marked $(*)$, if only a limited set of columns are required, only those columns must be computed, though the entire vector $\pi$ must be computed. For example, if only column $j \leq n-1$ is required, then only column $j$ must be computed in the steps marked $(*)$, and we do not need the entire vector $\mathbf{t}$, but only its $j$-th entry $t_{j}=\frac{1}{\mathbf{u}^{T} \mathbf{u}} \mathbf{u}_{1}^{T}\left[A_{11}^{-1}\right]_{: j}$, obtainable from the $j$-th column of $A_{11}^{-1}=\left(\widetilde{\mathbf{L}}_{11}\right)^{-1}$ already computed. In any case step 6 requires the solution of an extra system of linear equations for $\mathbf{w}$ (14):

$$
\mathbf{w}=\frac{1}{\mathbf{v}^{T} \mathbf{v}}\left(\widetilde{\mathbf{L}}_{11}\right)^{-1} \mathbf{v}_{1}=\frac{1}{\mathbf{v}^{T} \mathbf{v}}\left(\operatorname{Diag}\left(\mathbf{x}_{1: n-1}\right)\right) \boldsymbol{\Pi}_{1}^{-1 / 2}\left(\mathbf{L}_{11}^{\mathrm{d}}\right)^{-1} \mathbf{z}_{1}
$$

where $\mathbf{z}_{1} \stackrel{\text { def }}{=} \boldsymbol{\Pi}_{1}^{1 / 2} \widehat{\mathbf{D}}_{1}^{-1} \mathbf{v}_{1}$. Using (7), the boxed expression can be computed as follows:

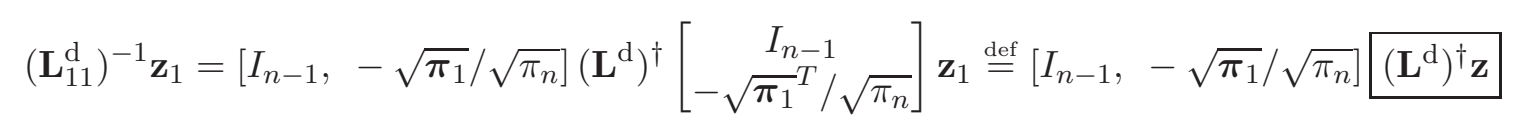

where $\mathbf{z}^{T} \stackrel{\text { def }}{=}\left[\mathbf{z}_{1}^{T},-\frac{{\sqrt{\pi_{1}}}^{T} \mathbf{z}_{1}}{\sqrt{\pi_{n}}}\right]$. The expression $\left(\mathbf{L}^{\mathrm{d}}\right)^{\dagger} \mathbf{z}$ can be computed as in Algorithm 1 step 3 using the same restarted GMRES procedure, based on the identity (9).

\section{Performance.}

To illustrate how the theoretical complexity corresponds to practice we generate a sequence of synthetic graphs using preferential attachment [1] with $2 n$ edges plus an extra set of $n$ randomly placed one-way edges to make the graph a digraph for a total of $3 n$ edges. Table 1 shows the time to compute the stationary probabilities $\boldsymbol{\pi}$ and the time to solve a single linear system involving $\mathbf{L}^{\mathrm{d}}$ using restarted GMRES. We run the methods in their original unpreconditioned form to show the correspondence between the theoretical complexity bounds and the numerical behavior in practice. We also show the number of matrix-vector products, which is solely a function of the number of overall iterations, which in turn is entirely dependent on the eigenvalue distribution of Laplacian. This, of course, depends on the nature of the underlying graph and would have to be analysed on a case-by-case basis. In the sequence of synthetic graphs constructed for this illustration, it is seen that the number of iterations is a slowly growing function of the dimension. Except for the modest increase in the number of matrix-vector products, the cost of the methods approximately double when the dimension $n$ is doubled. Here, the iterative methods were applied with a zero tolerance of $10^{-9}$. Using Matlab R2018a on a 2.5GHz Linux desktop with 8 Intel(R) Core(TM) i7-7700 CPU cores and 32 GB RAM, each experiment was repeated 4 times with averages shown in Table 1 .

The computation requires the storage of the adjacency matrix in sparse format plus up to $\ell$ auxiliary vectors of length $n$ and an $\ell \times \ell$ matrix, where $\ell$ is a user parameter independent of $n$. The off-the-shelf methods to compute the pseudo-inverse would use direct methods: solving the homogeneous system (10) for the vector of stationary probabilities, and using Theorem 1(c) or 4(c) to solve for the pseudo-inverse given the left and right annihilating vectors $\mathbf{u}, \mathbf{v}$. In both cases, the process would involve solving a nonsymmetric system of linear equations using a method like Gaussian elimination with partial pivoting. This 
Table 1: Performance on synthetic graphs \& one social network

\begin{tabular}{|c|r|r|r|r|}
\hline \multicolumn{1}{|c|}{$n$} & \multicolumn{2}{|c|}{ get $\boldsymbol{\pi}$} & \multicolumn{1}{c|}{ GMRES $(1 \mathrm{col})$} \\
\hline \multicolumn{1}{c|}{ dim } & $\# M v$ & time $(\mathrm{ms})$ & $\# \mathbf{~} \boldsymbol{v}$ & time $(\mathrm{ms})$ \\
\hline 1024 & 237 & 3.919 & 59 & 7.097 \\
2048 & 286 & 5.297 & 65 & 9.331 \\
4096 & 303 & 11.685 & 68 & 23.451 \\
8192 & 369 & 28.223 & 74 & 51.270 \\
16384 & 429 & 56.804 & 82 & 82.047 \\
32768 & 347 & 88.941 & 77 & 135.895 \\
65536 & 391 & 192.741 & 83 & 265.114 \\
131072 & 429 & 427.461 & 86 & 483.605 \\
262144 & 528 & 1130.698 & 95 & 1136.207 \\
\hline \hline \multicolumn{5}{|c|}{ Epinions data set } \\
\hline 75889 & 682 & 477.362 & 68 & 304.657 \\
\hline
\end{tabular}

would require $O\left(n^{3}\right)$ work with $O\left(n^{2}\right)$ storage, even if solving for just one column. For example, the largest case shown $\left(n=2^{18}\right)$ in the table would require $n^{2} \cdot 8 \approx 550 \mathrm{~GB}$ space, while the sparse iterative methods proposed here required only $8 \cdot(n \cdot \mathrm{d} \cdot n \ell+1 . o . t) \approx 71 \mathrm{MB}$, where we use $\mathrm{d}=4=1+$ \#edges_per_vertex, $\ell=30$, and 8 bytes per double word. Results using purely direct methods were reported in [7], where an off-the-shelf minimum degree ordering was used to reduce the fill in exact Gaussian elimination, but the induced fill was still observed to be $O\left(n^{2}\right)$ for the randomaly generated synthetic graphs. One could trade off accuracy for the direct methods by fixing the ordering of the equations to reduce fill, thereby substantially reducing the cost as proposed in [10, 11]. However, here we avoid the issue of fill-in entirely by using purely iterative methods with guarantees on the progress made at each iteration toward the solution. One can iterate to reduce the error to any desired tolerance within the range of the underlying arithmetic precision, without adding to the memory footprint.

Table 1 also shows performance on an Epinions data set [32], augmented with an evaporating node with an evaporating probability of $\gamma=0.05$ followed by a uniformly random restart (akin to the pagerank teleportation probability [31]). In other words, at every transition the walker has a 5\% chance of "evaporating" to the extra node, and from the extra node the walker transitions to one of the original nodes with equal probability. Including the extra node and associated links and 9 old nodes not otherwise connected to any other old node, the resulting graph has 75,889 nodes and 660,613 links.

\section{Discussion and Conclusions.}

We have used several off-the-shelf matrix iterative methods to compute individual columns of the pseudo-inverse of a digraph Laplacian matrix in time linear in the number of edges times a factor depending on how difficult it is to cut the graph into separate connected components. The full pseudo-inverse can be computed in time that is amortized to almost constant time per matrix entry. The methods proposed here depend exclusively on iterative methods and do not make any use of an elimination scheme that results in fill-ins, unlike methods using a variant of Gaussian elimination. They are relatively efficient and enjoy a plethora of available implementations.

The overall complexity is entirely dependent on the convergence rates for the iterative eigensolver and GMRES, which in turn depend on the smallest nonzero eigenvalues of $\mathbf{I}-\mathbf{P}$ and $\mathbf{L}+\mathbf{L}^{T}$, respectively. The former is related to the mixing rate of the random walk, while the latter is closely related to the Cheeger constant [8]. In both cases a tiny eigenvalue corresponds to a graph that can be split with a small cut. The 
convergence of any iterative method would depend on similar quantities in some fashion.

\section{Appendix}

We collect in this Appendix some results from the literature on which this paper is based.

9. Compute Stationary Probabilities. The vector of stationary probabilities $\boldsymbol{\pi}$ is the eigenvector of $\mathbf{P}^{T}$ corresponding to the eigenvalue $\lambda=1$. Since the underlying graph is strongly connected, the Perron Frobenius theory guarantees eigenvalue $\lambda=1$ is simple. The number of other eigenvalues of modulus 1 is equal to the periodicity of the graph or random walk. For instance, a bipartite graph will have an eigenvalue -1 . If per is the period of the graph and we use $\ell>$ per vectors in the following algorithm then the algorithm is guaranteed to converge at a rate bounded by $\left|\lambda_{\ell+1}(P)\right|<1$ [37] since $\lambda=1$ is known and is a simple eigenvalue of largest modulus.

Algorithm 3. Modified Subspace Iteration. [37]

Input: matrix $A$, hyperparameters: tol, initial guess $X^{[0]} \in \mathbb{R}^{n \times \ell}$.

Output: $\mathbf{z}=$ eigenvalue corresponding to eigenvalue 1 .

1. Set $Z=$ orthogonalize $\left(X^{[0]}\right)$, where $Z_{:, 1}$ is all non-negative.

2. Repeat until convergence:

(i) Set $Q=$ orthogonalize $(A Z)$.

(ii) Compute $\ell \times \ell$ Schur Decomposition $\left[U T U^{T}\right]=Q^{T} A Q$ with the diagonal entries of $T$ ordered to put the entry closest to 1 in the 1,1 position.

(iii) Set $Z=Q U$. Ensure the first column $Z_{:, 1}$ is all non-negative (flipping signs of rows of $Z$ to make the first column all non-negative, if necessary).

3. Return $\mathbf{z}=Z_{:, 1}$.

The cost per iteration is $\operatorname{cost}_{M V} \cdot(\ell+1)+c_{5}\left(n \ell^{2}\right)+c_{6}\left(\ell^{3}\right)$ where the first term accounts for the matrix vector products, the second term accounts for the orthogonalization (Alg 3 step 2(i)) and the third term accounts for the $\ell \times \ell$ Schur decomposition (step 2(ii)). Here $M v$ is the number of matrix vector products. Each matrix-vector product requires $\operatorname{cost}_{M_{V}}=2 \cdot \mathrm{nnz}$ (matrix) flops (one multiply and one add per matrix entry). Here each matrix entry corresponds exactly to an edge in the graph. So the total cost per iteration is

$$
\operatorname{cost}_{1}^{\text {subspace }}=\underbrace{c_{2} \text { \#nonzeros } \cdot(\ell+1)}_{\text {cost }_{M_{V}}}+\underbrace{c_{3} n \ell^{2}}_{\text {orthogonalization }}+\underbrace{c_{4} \ell^{3}}_{\text {Schur decomp }},
$$

for some small constants $c_{2}, c_{3}, c_{4}$ at most 10 . The storage required is (in words)

$$
\text { space }^{\text {subspace }}=\underbrace{(\# \text { nonzeros }+n)}_{\text {sparse } A}+\underbrace{2(n \ell)}_{\text {iterates } Z, Q}+\underbrace{O\left(\ell^{2}\right)}_{\text {temporaries } U, T}
$$

10. Restarted GMRES. The heart of the computation of the pseudo-inverse is the use of Theorem 1 (a) to convert a pseudo-inverse computation to an ordinary inverse computation. The restarted GMRES algorithm has received much attention in the literature (see [34] and references therein) with many enhancements for numerical stability that do not impact the cost by more than a constant factor. For the purposes of showing 
the overall cost of the algorithm, we show a simplified sketch of the basic algorithm. By using restarted GMRES, as opposed to ordinary GMRES, we bound the cost of each iteration and the memory footprint.

\section{Algorithm 4. Arnoldi-based Restarted GMRES.}

Input: Matrix $A$, right hand side $\mathbf{b}$, hyperparameters: restart count $\ell$, outer iteration limit maxit, tolerance tol, initial vector $\mathrm{x}^{[0]}$.

Output: solution $\mathrm{x}$ such that $\|\mathbf{r}\|=\|A \mathbf{x}-\mathbf{b}\|<$ tol.

1. Compute $\mathbf{r}^{[0]}=\mathbf{b}-A \mathbf{x}^{[0]}$

2. For $k=0,1,2, \ldots$ maxit:

(i) Set $\beta=\left\|\mathbf{r}^{[k]}\right\|_{2}$ and set $\mathbf{v}_{1}=\mathbf{r}^{[k]} / \beta$.

(ii) If $\beta<$ tol, return solution $\mathbf{x}=\mathbf{x}^{[k]}$.

(iii) Generate orthonormal Arnoldi basis $V_{\ell+1}=\left[\mathbf{v}_{1}, \cdots, \mathbf{v}_{\ell+1}\right]$ for the Krylov space $\operatorname{span}\left\{\mathbf{r}_{0}, A \mathbf{r}_{0}, \ldots, A^{l} \mathbf{r}_{0}\right\}$, and upper Hessenberg matrix $\bar{H}_{\ell} \in \mathbb{R}^{\ell+1 \times \ell}$ such that $A V_{\ell}=V_{\ell+1} \bar{H}_{\ell}$.

(iv) Compute $\mathbf{y}^{[k]}=\arg \min _{\mathbf{y}}\left\|\beta \mathbf{e}_{1}-\bar{H}_{\ell} \mathbf{y}\right\|_{2}^{2}$.

(v) Set $\mathbf{x}^{[k+1]}=\mathbf{x}^{[k]}+V_{\ell} \mathbf{y}^{[k]}$

The cost of one outer step2] of restarted GMRES is $\ell \cdot \operatorname{cost}_{M v}+O\left(n \ell^{2}+\ell^{2}\right)$ [34]. Here $\operatorname{cost}_{M v}$ is the cost of one matrix vector product involving sparse matrix $A$. This takes one floating multiply and one floating add for each nonzero matrix element. So the cost is $\operatorname{cost}_{M v}=O(\mathrm{nnz}(A))$. The Arnoldi step 2(iii) has one matrix vector product and an orthogonalization step for each of the $\ell$ Krylov vectors generated [34]. Step 2(iv) is an $(\ell+1) \times \ell$ least squares problem costing $O\left(\ell^{2}\right)$ to solve, due to the special Hessenberg structure of $\bar{H}$. The number of outer iterations required is controlled by the eigen-structure of the symmetric part $S(A)=\left(A+A^{T}\right) / 2$, which is related to the separability of the underlying graph [8]. Hence the total cost of restarted GMRES is

$$
\begin{aligned}
& \cos ^{\mathrm{GMRES}}=c^{\mathrm{g}}\left(\ell \cdot \cos _{\mathrm{MV}_{\mathrm{V}}}+c_{7}\left(n \ell^{2}+\ell^{2}\right)\right) \\
& =\underbrace{\frac{|\log (\mathrm{tol})|+\left|\log \left\|\mathbf{r}_{0}\right\|\right|}{|\log \sigma|}}_{\text {\#iterations }}(\underbrace{\ell \cdot \operatorname{cost}_{M v}}_{\text {Mat*vec }}+c_{7}(\underbrace{n \ell^{2}}_{\text {orthogonalization }}+\underbrace{\ell^{2}}_{\text {work with } H}))
\end{aligned}
$$

where $\sigma=\left(1-\frac{\left(\lambda_{\min }[S(A)]\right)^{2}}{\|A\|_{2}^{2}}\right)^{\ell / 2}$ and $c^{\mathrm{g}}=\frac{|\log (\mathrm{tol})|+\left|\log \left\|\mathbf{r}_{0}\right\|\right|}{|\log \sigma|}$. The storage required is (in words)

$$
\text { space }^{\text {GMRES }}=\underbrace{(\# \text { nonzeros }+n)}_{\text {sparse } A}+\underbrace{2(n \ell)}_{\text {Krylov vectors } V}+\underbrace{O\left(\ell^{2}\right)}_{\text {temporaries } H, Y} \text {. }
$$

Regarding the number of GMRES iterations, we have the following bound which yields Corollary 3 as an immediate consequence.

Theorem 5. [12, 13, 22, 26], Let $A$ be a matrix such that $S(A) \stackrel{\text { def }}{=}\left(A+A^{H}\right) / 2$ is Hermitian positive definite and let $\lambda_{\min }[S(A)]>0$ denote the smallest eigenvalue for $S(A)$. The residual $\mathbf{r}_{k}$ obtained by GMRES [35] after $k$ steps applied to the linear system $A \mathbf{x}=b$ satisfies

$$
\frac{\left\|\mathbf{r}_{k}\right\|_{2}}{\left\|\mathbf{r}_{0}\right\|_{2}} \leq\left(1-\frac{\left(\lambda_{\min }[S(A)]\right)^{2}}{\|A\|_{2}^{2}}\right)^{k / 2}
$$

To be self-contained, we give a sketch of a proof for this essential bound, referring to to [12, 13, 22, 26] for detailed proofs for this and several tighter bounds. First we need the following Lemma 
Lemma 6. Let $A \in \mathbb{C}^{n \times n}$ such that $S(A)=\left(A+A^{H}\right) / 2$ is positive definite, and let $\mathbf{v} \in \mathbb{C}^{n}$ with $\|\mathbf{v}\|_{2}=1$ be given. Let $\alpha_{\mathbf{v}}=\left[(A \mathbf{v})^{H} \mathbf{v}\right] /\left[(A \mathbf{v})^{H}(A \mathbf{v})\right]$. Then

$$
\left\|\mathbf{v}-\alpha_{\mathbf{v}} A \mathbf{v}\right\|^{2} \leq 1-\lambda_{\min }\left[S\left(A^{-1}\right)\right] \cdot \lambda_{\min }[S(A)] \leq 1-\frac{\left(\lambda_{\min }[S(A)]\right)^{2}}{\|A\|^{2}}
$$

Proof. $\alpha_{\mathbf{v}}$ is the value achieving the minimum in the scalar least squares problem $\min _{\alpha}\|\mathbf{v}-\alpha A \mathbf{v}\|_{2}^{2}$ and hence satisfies the Galerkin condition $(A \mathbf{v})^{H}\left(\mathbf{v}-\alpha_{\mathbf{v}} A \mathbf{v}\right)=0$. So we have

$$
\|\mathbf{v}-\alpha A \mathbf{v}\|^{2}=\mathbf{v}^{H}\left(\mathbf{v}-\alpha_{\mathbf{v}} A \mathbf{v}\right)=1-\alpha_{\mathbf{v}} \cdot \mathbf{v}^{H} A \mathbf{v}=1-\frac{\mathbf{w}^{H} A^{-1} \mathbf{w}}{\mathbf{w}^{H} \mathbf{w}} \cdot \mathbf{v}^{H} A \mathbf{v},
$$

where $\mathbf{w}=A \mathbf{v}$. A well known result on field of values for any matrix $M$ whose Hermitian part $S(M)=$ $\left(M+M^{H}\right) / 2$ is positive definite is the inequality [21] for any $\mathbf{x} \neq 0$

$$
\left|\frac{\mathbf{x}^{H} M \mathbf{x}}{\mathbf{x}^{H} \mathbf{x}}\right| \geq \operatorname{Re}\left(\frac{\mathbf{x}^{H} M \mathbf{x}}{\mathbf{x}^{H} \mathbf{x}}\right) \geq \lambda_{\min }[S(M)]>0 .
$$

Hence the first inequality (17) follows. The remaining inequality follows from the identity

$$
S\left(A^{-1}\right)=A^{-1} \cdot S(A) \cdot A^{-H} .
$$

Inverting both sides and taking norms yields

$$
\frac{1}{\lambda_{\min }\left[S\left(A^{-1}\right)\right]}=\left\|\left[S\left(A^{-1}\right)\right]^{-1}\right\|_{2} \leq\|A\|_{2}^{2}\left\|[S(A)]^{-1}\right\|_{2}=\frac{\|A\|_{2}^{2}}{\lambda_{\min }[S(A)]}
$$

Sketch of Proof of Theorem 5. We include a proof sketch stripped down to its bare essentials. GMRES on a matrix $A$ with initial residual $\mathbf{r}_{0}$ will find in $k$ steps a solution with a residual $\mathbf{r}_{k}$ satisfying $\left\|\mathbf{r}_{k}\right\|=\min _{p \in \mathbb{P}_{k}}\left\|p(A) \mathbf{r}_{0}\right\|$, where $\mathbb{P}_{k}$ is the set of all polynomials $p$ of degree up to $k$ satisfying $p(0)=1$. In particular, after a single step $\left\|\mathbf{r}_{1}\right\|=\min _{p \in \mathbb{P}_{\nVdash}}\left\|p(A) \mathbf{r}_{0}\right\| \leq\left\|\left(I-\alpha\left(\mathbf{r}_{0}\right)\right) A \mathbf{r}_{0}\right\|$, where $\alpha\left(\mathbf{r}_{0}\right)=\left[\left(A \mathbf{r}_{0}\right)^{H} \mathbf{r}_{0}\right] /\left[\left(A \mathbf{r}_{0}\right)^{H}\left(A \mathbf{r}_{0}\right)\right]$. Hence we have the bound from Lemma 6 ; $\left\|\mathbf{r}_{1}\right\|_{2}^{2} \leq\left\|\mathbf{r}_{0}\right\|_{2}^{2} \cdot[1-$ $\lambda_{\min } S(A) \lambda_{\min } S\left(A^{-1}\right)$ ]. This amounts to a single step of a variant of the classical Richardson iteration. Repeating this Richardson iteration yields

$$
\mathbf{r}_{k}^{[\text {rich }]}=\left(I-\alpha\left(\mathbf{r}_{k-1}^{[\text {rich }]}\right) A\right) \cdots\left(I-\alpha\left(\mathbf{r}_{1}^{[\text {rich }]}\right) A\right)\left(I-\alpha\left(\mathbf{r}_{0}\right) A\right) \mathbf{r}_{0} .
$$

The norm of the residual after $k$ Richardson steps is bounded above by the convergence bound and bounded below by the norm of the GMRES residual after $k$ steps:

$$
\left\|\mathbf{r}_{k}^{[\text {GMRES }]}\right\|_{2}^{2}=\min _{p \in \mathbb{P}_{k}}\left\|p(A) \mathbf{r}_{0}\right\|_{2}^{2} \leq\left\|\mathbf{r}_{k}^{[\mathrm{rich}]}\right\|_{2}^{2} \leq\left(1-\lambda_{\min }[S(A)] \lambda_{\min }\left[S\left(A^{-1}\right)\right]\right)^{k}
$$

\section{Acknowledgements}

This research was supported in part by NSF grants 1835530 and 1922512. The author would like to thank the anonymous reviewer for many helpful comments that greatly improved the manuscript. 


\section{References}

[1] R. Albert and A.-L. Barabási. Statistical mechanics of complex networks. Rev. Mod. Phys., 74:47-97, Jan 2002.

[2] D. Aldous and J. A. Fill. Reversible Markov chains and random walks on graphs, 2002. Unfinished monograph, recompiled 2014, available at http://www.stat.berkeley.edu/ users/aldous/RWG/book.html.

[3] A. Berman and R. J. Plemmons. Nonnegative Matrices in the Mathematical Sciences. Academic Press, New York, 1979. Reprinted by SIAM, Philadelphia, 1994.

[4] D. Boley, A. Buendia, and G. Golnari. Random walk Laplacian and network centrality measures. arxiv.org/abs/1808.02912, 2018.

[5] D. Boley, G. Ranjan, and Z.-L. Zhang. Commute times for a directed graph using an asymmetric Laplacian. Lin. Alg. \& Appl., 435:224-242, 2011.

[6] A. Buendia and D. Boley. Optimized graph-based trust mechanisms using hitting times. In AAMAS Intl Workshop on Trust in Agent Societies, May 2017.

[7] A. Buendia and D. Boley. Random walk fundamental tensor and graph importance measures. In 2019 Int'l Workshop on Big Social Media Data Management and Analysis BSMDMA2019 at IJCAI 2019, Aug. 2019.

[8] F. Chung. Laplacians and the Cheeger inequality for directed graphs. Annals of Combinatorics, 9(1):119, April 2005.

[9] F. R. K. Chung. Spectral Graph Theory. American Mathematical Society, 1997.

[10] M. B. Cohen, J. Kelner, R. Kyng, J. Peebles, R. Peng, A. B. Rao, and A. Sidford. Solving directed Laplacian systems in nearly-linear time through sparse LU factorizations. arxiv.org/abs/1811.10722, 2018.

[11] M. B. Cohen, J. Kelner, J. Peebles, R. Peng, A. Sidford, and A. Vladu. Faster algorithms for computing the stationary distribution, simulating random walks, and more. In IEEE 57th Annual Symp. on Found. Comput. Sci. (FOCS), pages 583-592, Oct 2016.

[12] M. Eiermann and O. Ernst. Geometric aspects of the theory of Krylov subspace methods,. Acta Numer, 10:251-312, 2001.

[13] H. C. Elman. Iterative methods for large sparse nonsymmetric systems of linear equations. PhD thesis, Yale University, New Haven, 1982.

[14] F. Fouss, A. Pirotte, J.-M. Renders, and M. Saerens. Random-walk computation of similarities between nodes of a graph with application to collaborative recommendation. IEEE Transactions on Knowledge and Data Engineering, 19(3):355-369, 2007.

[15] F. R. Gantmacher. Theory of Matrices, volume 2. Chelsea, New York, 1959. 
[16] D. Gleich, L. Zhukov, and P. Berkhin. Fast parallel PageRank: A linear system approach. In $W W W$ 2005, 2005.

[17] G. Golnari, A. Asiaee, A. Banerjee, and Z.-L. Zhang. Revisiting non-progressive influence models: Scalable influence maximization. In UAI, pages 316-325, 2015.

[18] G. Golnari, Z.-L. Zhang, and D. Boley. Markov fundamental tensor and its applications to network analysis. Linear Algebra and Appl., 564:126-158, 2019.

[19] G. H. Golub and C. F. Van Loan. Matrix Computations. Johns Hopkins Univ. Press, 4th edition, 2013.

[20] C. M. Grinstead and J. L. Snell. Introduction to Probability. American Mathematical Society, 2nd edition, 2006. www. dartmouth. edu/ chance/teaching_aids/books_articles/ probability_book/book.html.

[21] R. A. Horn and C. R. Johnson. Topics in Matrix Analysis. Cambridge University Press, Cambridge, 1991.

[22] W. Joubert. On the convergence behavior of the restarted GMRES algorithm for solving nonsymmetric linear systems. Num. Lin. Alg. Appl., 1(5):427-447, 1994.

[23] J. Kemeny and J. Snell. Finite Markov Chains. Springer-Verlag, 1976.

[24] Y. Li and Z.-L. Zhang. Random walks on digraphs: A theoretical framework for estimating transmission costs in wireless routing. In The 29th IEEE Conference on Computer Communications (IEEE INFOCOM 2010), March 2010.

[25] Y. Li and Z.-L. Zhang. Random walks on digraphs, the generalized digraph Laplacian and the degree of asymmetry. In 7th Workshop on Algorithms and Models for Webgraphs WAW'10, Dec 13-17 2010. (colocated with WINE'10), wWw. cs.umn.edu/ zhzhang/Papers/Yanhua-Li-WAW10.pdf.

[26] J. Liesen and P. Tichý. The field of values bound on ideal GMRES. arxiv • org/abs/1211.5969, 2012.

[27] B. Liu, D. Parkes, and S. Seuken. Personalized hitting time for informative trust mechanisms despite sybils. In Int'l Conf. Auto. Agents \& Multiagent Sys. (AAMAS), 2016.

[28] C. Meyer. Generalized inversion of modified matrices. SIAM J. Appl. Math., 24:315-323, 1973.

[29] C. Meyer. The role of the group generalized inverse in the theory of finite Markov chains. SIAM Rev., 17:443-464, 1975.

[30] J. Norris. Markov Chains. Cambridge Univ. Press, 1997.

[31] L. Page, S. Brin, R. Motwani, and T. Winograd. The pagerank citation ranking: Bringing order to the web. TR SIDL-WP-1999-0120, Computer Systems Laboratory, Stanford Univ., 1998. ilpubs.stanford.edu: $8090 / 422 /$.

[32] M. Richardson, R. Agrawal, and P. Domingos. Trust management for the semantic web. In ISWC, 2003. (Data from https://snap.stanford.edu/data/soc-Epinions1.html). 
[33] P. Robert. On the group-inverse of a linear transformation. J of Math Anal and Appl, 22:658-669, 1968.

[34] Y. Saad. Iterative Methods for Sparse Linear Systems. SIAM, 2nd edition, 2003.

[35] Y. Saad and M. Schultz. GMRES: A generalized minimal residual algorithm for solving nonsymmetric linear systems. SIAM J. Sci. and Stat. Comput., 7:856-869, 1986.

[36] D. A. Spielman and S.-H. Teng. Nearly linear time algorithms for preconditioning and solving symmetric, diagonally dominant linear systems. SIAM J Matrix Anal, 35:835-885, 2014.

[37] G. W. Stewart. Simultaneous iteration for computing invariant subspaces of non-Hermitian matrices. Numer. Math., 25:123-136, 1976.

[38] D. Zhou, J. Huang, and B. Schölkopf. Learning from labeled and unlabeled data on a directed graph. In Proc. 22nd Int'l Conf. Machine Learning, pages 1041-1048, 2005. 